\title{
Dynamic Analysis of an SEIR Model with Distinct Incidence for Exposed and Infectives
}

\author{
Junhong Li and Ning Cui \\ Department of Mathematics and Sciences, Hebei Institute of Architecture and Civil Engineering, Zhangjiakou, Hebei 075000, China \\ Correspondence should be addressed to Junhong Li; jhli2011@163.com
}

Received 17 April 2013; Accepted 9 May 2013

Academic Editors: J. Banaś and M. M. Cavalcanti

Copyright (C) $2013 \mathrm{~J}$. Li and N. Cui. This is an open access article distributed under the Creative Commons Attribution License, which permits unrestricted use, distribution, and reproduction in any medium, provided the original work is properly cited.

\begin{abstract}
An SEIR model with vaccination strategy that incorporates distinct incidence rates for the exposed and the infected populations is studied. By means of Lyapunov function and LaSalle's invariant set theorem, we proved the global asymptotical stable results of the disease-free equilibrium. The sufficient conditions for the global stability of the endemic equilibrium are obtained using the compound matrix theory. Furthermore, the method of direct numerical simulation of the system shows that there is a periodic solution, when the system has three equilibrium points.
\end{abstract}

\section{Introduction}

Mathematical models have become important tools in analyzing the spread and the control of infectious diseases. Many infectious diseases in nature, such as measles, HIV/AIDS, SARS, and tuberculosis (see [1-6]), incubate inside the hosts for a period of time before the hosts become infectious. Li and Fang (see [7]) studied the global stability of an age-structured SEIR model with infectivity in latent period. Yi et al. (see [8]) discussed the dynamical behaviors of an SEIR epidemic system with nonlinear transmission rate. $\mathrm{Li}$ and Zhou (see [9]) considered the global stability of an SEIR model with vertical transmission and saturating contact rate.

In this paper, we will consider an SEIR model that the diseases can be infected in the latent period and the infected period. The population size $N$ is divided into four homogeneous classes: the susceptible $S(t)$, the exposed (in the latent period) $E(t)$, the infective $I(t)$, and the recovered $R(t)$. It is assumed that all the offsprings at birth are susceptible to the disease. The inflow rate (including birth and immigration) and outflow rate (including natural death and emigration) are denoted by $b$ and $d$, respectively. The rate of disease-caused death is taken as $\alpha$. We assume that susceptible individuals are vaccinated at a constant per capita rate $a$. Due to the partial efficiency of the vaccine, only $\sigma$ fraction of the vaccinated susceptibles goes to the recovered class. The remained $1-\sigma$ fraction of the vaccinated susceptibles has no immunity at all and goes to the exposed class after infected by contact with the infectives. If $\sigma=0$, it means that the vaccine has no effect at all, and if $\sigma=1$, the vaccine is perfectly effective. The positive parameter $\varepsilon$ is the rate at which the exposed individuals become infectious. $\gamma$ is the constant rate, at which the infectious individuals recover with acquiring permanent immunity. The transfer mechanism from the class $S(t)$ to the class $E(t)$ is guided by the function $\beta(I+q E) / N$, where $\beta$ is the force of infection. $q$ denotes the relative measure of infectiousness for the asymptomatic class $E(t)$.

Based on these considerations, and with reference to [1012], the SEIR model is given by the following system of differential equations:

$$
\begin{gathered}
S^{\prime}=b N-F(S, E, I)-(\sigma a+d) S, \\
E^{\prime}=F(S, E, I)-(d+\varepsilon) E, \\
I^{\prime}=\varepsilon E-(\alpha+\gamma+d) I, \\
R^{\prime}=\sigma a S+\gamma I-d R, \\
0=S+E+I+R-N,
\end{gathered}
$$

where the derivative $d / d t$ is denoted by ' and $F(S, E, I)=$ $\beta S(1-a)(I+q E) / N+\beta a S(1-\sigma)(I+q E) / N$.

Thus, the total population size $N$ implies $N^{\prime}=(b-d) N-$ $\alpha I$. Let $s=S / N, e=E / N, i=I / N$, and $r=R / N$. 
Because the variable $R$ does not appear in the equations of $S, E$, and $I$, we only need to consider the following subsystem:

$$
\begin{gathered}
s^{\prime}=b-\beta s(1-\sigma a)(i+q e)-(\sigma a+b) s+s \alpha i, \\
e^{\prime}=\beta s(1-\sigma a)(i+q e)-(b+\varepsilon) e+e \alpha i, \\
i^{\prime}=\varepsilon e-(\alpha+\gamma+b) i+\alpha i^{2}, \\
0=s+e+i+r-1 .
\end{gathered}
$$

The system (2) is equivalent to (1). From biological considerations, we study (2) in the following closed set:

$$
T=\left\{(s, e, i) \in R_{+}^{3} \mid 0 \leq s+e+i \leq 1\right\},
$$

where $R_{+}^{3}$ denotes the nonnegative cone of $R^{3}$ including its lower dimensional faces.

\section{Equilibria and Global Stability}

It is easy to visualize that (2) always has a disease-free equilibrium $P_{0}(b /(\sigma a+b), 0,0)$. The Jacobian matrix of (2) at an arbitrary point $P(s, e, i)$ takes the following form:

$$
J(P)=\left[\begin{array}{ccc}
J_{11} & -q \beta(1-\sigma a) s & J_{13} \\
J_{21} & J_{22} & J_{23} \\
0 & \varepsilon & J_{33}
\end{array}\right],
$$

where

$$
\begin{gathered}
J_{11}=-\beta(1-\sigma a)(i+q e)-(\sigma a+b)+\alpha i, \\
J_{13}=\alpha s-\beta(1-\sigma a) s, \\
J_{23}=\alpha e+\beta(1-\sigma a) s, \\
J_{33}=2 \alpha i-(\alpha+\gamma+b), \\
J_{22}=\beta q(1-\sigma a) s-(b+\varepsilon)+\alpha i, \\
J_{21}=\beta(1-\sigma a)(i+q e) .
\end{gathered}
$$

Theorem 1. If $R_{0}<1$, the disease-free equilibrium $P_{0}$ is locally asymptotically stable, where

$$
R_{0}=\frac{b \beta(1-\sigma a)(q \alpha+q \gamma+q b+\varepsilon)}{(b+\varepsilon)(\alpha+b+\gamma)(\sigma a+b)} .
$$

Proof. Let

$$
\lambda_{1}=\frac{(\varepsilon+q \alpha+q \gamma+q b)(\alpha+\gamma+2 b+\varepsilon)}{(\alpha+\gamma+b)(b+\varepsilon) q R_{0}}-1 .
$$

We calculate the characteristic equation of $J\left(P_{0}\right)$ as follows:

$$
\begin{aligned}
& (\lambda+\sigma a+b) \\
& \times\left(\lambda^{2}+\frac{b q \beta(1-\sigma a) \lambda_{1} \lambda}{(\sigma a+b)}\right. \\
& \left.\quad+(b+\varepsilon)(\alpha+\gamma+b)\left(1-R_{0}\right)\right)=0 .
\end{aligned}
$$

The stability of $P_{0}$ is equivalent to all eigenvalues of (8) being with negative real parts, which can be guaranteed by $R_{0}<1$. Consequently, the disease-free equilibrium is local asymptotical stability. This proves the theorem.

Theorem 2. If $R_{01} \leq 1$, the disease-free equilibrium $P_{0}$ is globally asymptotically stable, where

$$
R_{01}=\frac{\beta(1-\sigma a)(q \alpha+q \gamma+q b+\varepsilon)}{(b+\varepsilon)(\alpha+b+\gamma)} .
$$

Proof. Consider the following function:

$$
L=(\alpha+\gamma+b) e+\beta(1-\sigma a) i
$$

Its derivative along the solutions to the system (2) is as follows:

$$
\begin{aligned}
L^{\prime}= & i[(\alpha+\gamma+b) \beta(1-\sigma a) s-(1-\sigma a)(\alpha+\gamma+b-\alpha i)] \\
& +e[(\alpha+\gamma+b) q \beta(1-\sigma a) s \\
& \quad-(\alpha+\gamma+b)(b+\varepsilon+\alpha i)+(1-\sigma a) \varepsilon] \\
& \quad e(\alpha+\gamma+b)(b+\varepsilon)\left(R_{01}-1\right) \\
\leq & 0 .
\end{aligned}
$$

Furthermore, $L^{\prime}=0$ only if $e=0$. The maximum invariant set in $\left\{(s, e, i) \in T: L^{\prime}=0\right\}$ is the singleton $\left\{P_{0}\right\}$. When $R_{01} \leq 1$, the global stability of $P_{0}$ follows from LaSalle's invariance principle (see [13]). This completes the proof.

Theorem 3. Equation (2) has a unique endemic equilibrium $P^{*}\left(s^{*}, e^{*}, i^{*}\right)$ if $b \geq \alpha$ and $\Delta>1$, where

$$
\begin{aligned}
\Delta= & (b+\varepsilon-\alpha)\left(\frac{\gamma}{b}+1\right) \\
& \times\left(\frac{1}{\varepsilon}-\frac{(\sigma a+b-\alpha)}{\beta(1-\sigma a)(q b+q \gamma+\varepsilon)}\right) .
\end{aligned}
$$

Proof. Let the right side of each of the first three differential equations equal to zero in (2); we obtain the following:

$$
\begin{gathered}
\beta s^{*}(1-\sigma a)\left(i^{*}+q e^{*}\right)-(b+\varepsilon) e^{*}+\alpha e^{*} i^{*}=0, \\
\varepsilon e^{*}-(\alpha+\gamma+b) i^{*}+\alpha i^{* 2}=0, \\
b-\beta s^{*}(1-\sigma a)\left(i^{*}+q e^{*}\right)-(\sigma a+b) s^{*}+\alpha s^{*} i^{*}=0,
\end{gathered}
$$

with $s^{*}>0, e^{*}>0$ and $i^{*}>0$. So we get

$$
\begin{gathered}
\frac{b}{s^{*}}=\beta(1-\sigma a)\left(i^{*}+q e^{*}\right)-(\sigma a+b)+\alpha i^{*}, \\
\frac{\beta s^{*}(1-\sigma a)\left(i^{*}+q e^{*}\right)}{e^{*}}=(b+\varepsilon)+\alpha i^{*}, \\
\varepsilon e^{*}=(\alpha+\gamma+b) i^{*}-\alpha i^{* 2} .
\end{gathered}
$$


When the three equations of (14) are multiplied together, we obtain the following:

$$
\begin{aligned}
& b \beta \varepsilon(1-\sigma a)\left[\frac{(\alpha+\gamma+b) q}{\varepsilon}+1-\frac{\alpha q i^{*}}{\varepsilon}\right] \\
& =\left(b+\varepsilon-\alpha i^{*}\right)\left(\alpha+\gamma+b-\alpha i^{*}\right) \\
& \quad \times\left[\frac{-\alpha q \beta(1-\sigma a) i^{* 2}}{\varepsilon}\right. \\
& \quad+\left(\frac{\beta(1-\sigma a)(\alpha q+\gamma q+b q+\varepsilon)}{\varepsilon}+\alpha\right) i^{*} \\
& \quad-(\sigma a+b)] .
\end{aligned}
$$

Define the following:

$$
\begin{gathered}
f(i)=f_{1}(i)(b+\varepsilon-\alpha i)(\alpha+\gamma+b-\alpha i), \\
g(i)=b \beta \varepsilon(1-\sigma a)\left[\frac{(\alpha+\gamma+b) q}{\varepsilon}+1-\frac{\alpha q i}{\varepsilon}\right],
\end{gathered}
$$

where

$$
\begin{aligned}
f_{1}(i)= & {\left[\frac{-\alpha q \beta(1-\sigma a) i^{2}}{\varepsilon}\right.} \\
& +\left(\frac{\beta(1-\sigma a)(\alpha q+\gamma q+b q+\varepsilon)}{\varepsilon}+\alpha\right) i \\
& -(\sigma a+b)],
\end{aligned}
$$

and the roots of $f(i)$ are $i_{1}=(b+\varepsilon) / \alpha>1, i_{2}=(\alpha+\gamma+$ $b) / \alpha>1$, and the other two are $i_{3}, i_{4}$ which satisfy $f_{1}(i)=0$. $i_{0}=(\alpha+\gamma+b) / \alpha+\varepsilon /(\alpha q)$ is the root of $g(i)$. Direct calculations show the following:

$$
\begin{gathered}
g(1)=b \beta \varepsilon(1-\sigma a)(q b+q \gamma+\varepsilon)>0, \\
f(0)=-(\sigma a+b)(b+\varepsilon)(\alpha+\gamma+b)<0, \\
f(1)=\Delta g(1), \quad i_{0}>i_{1}, \quad i_{0}>i_{2}, \\
i_{3} i_{4}=\frac{\varepsilon(\sigma a+b)}{\alpha q \beta(1-\sigma a)}>0, \\
i_{3}+i_{4}=1+\frac{b+\gamma}{\alpha}+\varepsilon\left(\frac{1}{\alpha q}+\frac{1}{q \beta(1-\sigma a)}\right)>2 .
\end{gathered}
$$

Since $\Delta>1$, the linear function $g(i)$ has exactly one intersection with the function $f(i)$ where $i$ lies in the interval $(0,1)$. Furthermore, $s^{*}$ and $e^{*}$ can be uniquely determined from $i^{*}$ by the following:

$$
\begin{gathered}
e^{*}=\frac{i^{*}\left(\alpha+\gamma+b-\alpha i^{*}\right)}{\varepsilon}, \\
s^{*}=\frac{b}{\left[\beta(1-\sigma a)\left(i^{*}+e^{*}\right)+(\sigma a+b)+\alpha i^{*}\right]} .
\end{gathered}
$$

From this, we can easily see that (2) has a unique endemic equilibrium. This completes the proof.

Denote the interior of $T$ by $\stackrel{\circ}{T}$. In this paper, we obtain sufficient conditions that the equilibrium is globally asymptotically stable using the geometrical approach of $\mathrm{Li}$ and Muldowney in [14].

Theorem 4. The unique endemic equilibrium $P^{*}$ is globally asymptotically stable in $\stackrel{\circ}{T}$, when

$$
b \geq \alpha, \quad \Delta>1, \quad R_{01}>1, \quad \alpha<\min \{\sigma a, b, \varepsilon\} .
$$

Proof. Since $R_{01}>1$, namely, $\Delta<R_{0}$ and $P_{0}$ is unstable, we can easy see that $(2)$ satisfies the assumptions $\left(H_{1}\right)$ and $\left(\mathrm{H}_{2}\right)$ (see [14]) in the interior of its feasible region $T$. The unique equilibrium is locally asymptotically stable using simple calculation.

Let $x=(s, e, i)$ and $f(x)$ denotes the vector field of (2) and

$$
J^{[2]}(P)=\left(\begin{array}{ccc}
a_{11} & q \beta s(1-\sigma a)+\alpha e & -\alpha s+\beta s(1-\sigma a) \\
\varepsilon & a_{22} & -q \beta s(1-\sigma a) \\
0 & \beta(1-\sigma a)(i+q e) & a_{33}
\end{array}\right),
$$

where

$$
\begin{gathered}
a_{11}=-\beta(1-\sigma a)(i+q e-q s)-(\sigma a+2 b+\varepsilon)+2 \alpha i, \\
a_{22}=-\beta(1-\sigma a)(i+q e)-(2 b-d+\alpha+\beta)+3 \alpha i, \\
a_{33}=-q \beta s(1-\sigma a)-(2 b+\varepsilon+\alpha+\beta)+3 \alpha i .
\end{gathered}
$$

Set the the following function:

$$
P(x)=P(s, e, i)=\operatorname{diag}\left(1, \frac{e}{i}, \frac{e}{i}\right) .
$$

Then the matrix $B=P_{f} P^{-1}+P J^{[2]} P^{-1}$ can be written in block form as follow:

$$
B=\left(\begin{array}{ll}
B_{11} & B_{12} \\
B_{21} & B_{22}
\end{array}\right),
$$

where

$$
\begin{aligned}
& B_{11}=a_{11}, \quad B_{21}=\left(\frac{\varepsilon e}{i} 0\right)^{\prime}, \\
& B_{12}=\left(\frac{\beta(1-\sigma a) s i}{e}+\alpha i \frac{(\beta(1-\sigma a)-\alpha) s i}{e}\right) \text {, } \\
& B_{221}=\frac{e^{\prime}}{e}-\frac{i^{\prime}}{i}+a_{22}, \quad B_{222}=\frac{e^{\prime}}{e}-\frac{i^{\prime}}{i}+a_{33} \text {, } \\
& B_{22}=\left(\begin{array}{cc}
B_{221} & -q \beta s(1-\sigma a) \\
\beta(1-\sigma a)(i+q e) & B_{222}
\end{array}\right) .
\end{aligned}
$$

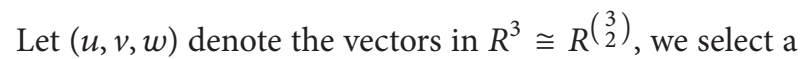
norm in $R^{3}$ as $|(u, v, w)|=\max \{|u|,|v|+|w|\}$ and let $\rho$ denote 


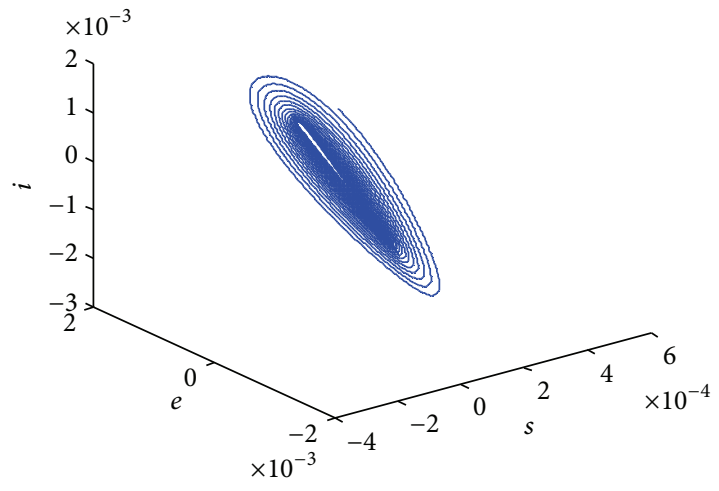

(a)

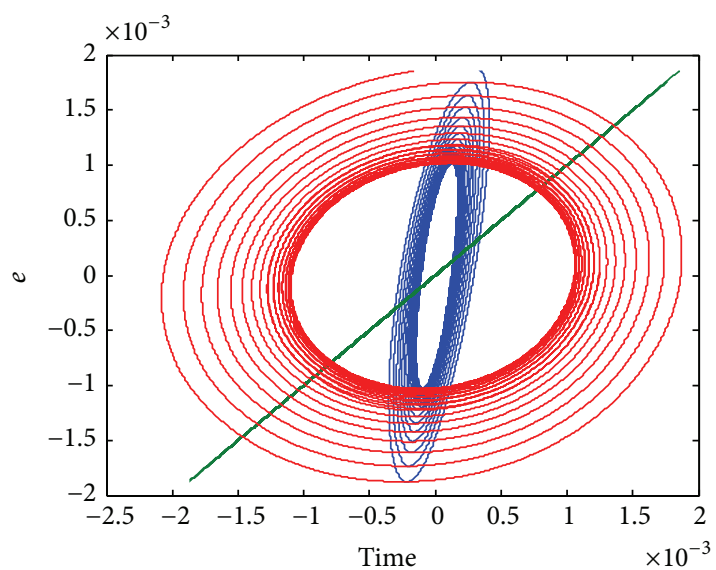

(c)

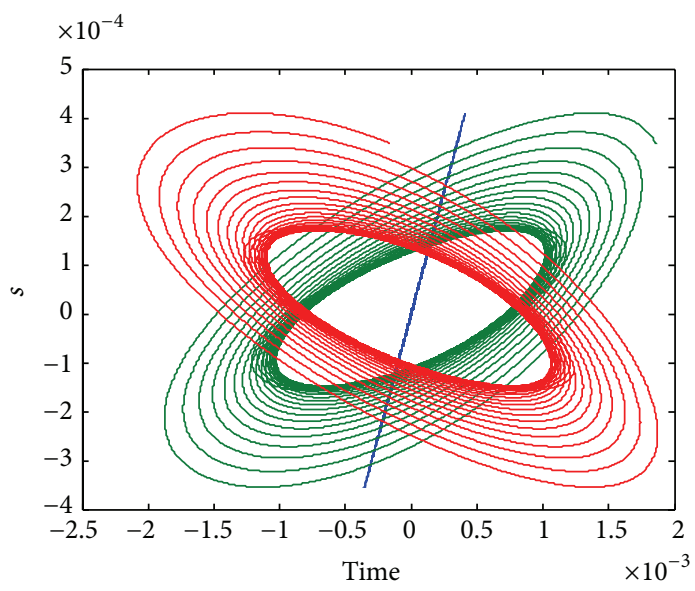

(b)

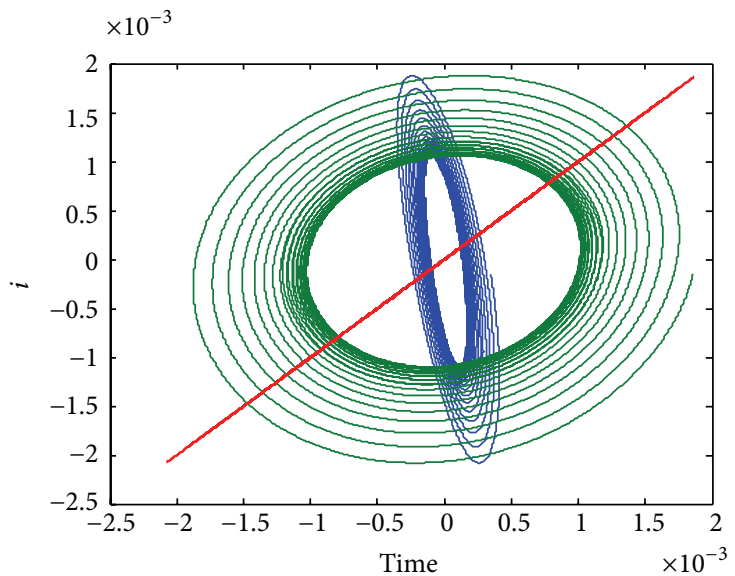

(d)

Figure 1: (a) Phase portraits of (2). (b) Time series of $s$. (c) Time series of $e$. (d) Time series of $i$.

the Lozinskii measure with respect to this norm. Using the method of estimating $\rho$ in [15], we have $\rho(B) \leq \sup \left(g_{1}, g_{2}\right)$, where

$$
g_{1}=\rho_{1}\left(B_{11}\right)+\left|B_{12}\right|, \quad g_{2}=\left|B_{21}\right|+\rho_{1}\left(B_{22}\right)
$$

$\left|B_{12}\right|,\left|B_{21}\right|$ are matrix norms with respect to the $l_{1}$ vector norm, and $\rho_{1}$ denotes the Lozinskii measure with respect to $l_{1}$ norm. More specifically, $\rho_{1}\left(B_{11}\right)=B_{11},\left|B_{21}\right|=\varepsilon e / i$, and $\left|B_{12}\right|=\beta(1-\sigma a) s i / e+\alpha i$.

Rewriting the system (2), we have the following:

$$
\frac{\varepsilon e}{i}=\frac{i^{\prime}}{i}+(\alpha+\gamma+b)-\alpha i
$$

Therefore,

$$
\begin{gathered}
g_{1}=\frac{e^{\prime}}{e}-\beta(1-\sigma a)(i+q e)-(\sigma a+b)+2 \alpha i, \\
g_{2}=\frac{e^{\prime}}{e}+2 \alpha i-b-\min \{\sigma a, \varepsilon\} .
\end{gathered}
$$

Since $b \geq \alpha$ and $\alpha<\min \{\sigma a, b, \varepsilon\}$, there is $m=\max \{\sigma a+b-$ $2 \alpha, b+\varepsilon-2 \alpha\}>0$ and

$$
\rho(B) \leq \sup \left(g_{1}, g_{2}\right) \leq \frac{e^{\prime}}{e}-m \text { for } t>\bar{t} .
$$

Along each solution $x\left(t, x_{0}\right)$ to (2) such that $x_{0} \in K$, where $K$ is the compact absorbing set, we thus have the following:

$$
\frac{1}{t} \int_{0}^{t} \rho(B) d s \leq \frac{1}{t} \log \frac{e(t)}{e(\bar{t})}+\frac{1}{t} \int_{0}^{\bar{t}} \rho(B) d s-m \frac{t-\bar{t}}{t},
$$

which implies

$$
\bar{q}_{2} \leq-\frac{m}{2}<0 .
$$

This completes the proof.

\section{Conclusion}

In this paper, we discuss an SEIR model that the diseases can be infected in the latent period and the infected period. 
The vaccine effectiveness is also taken into account. We investigate the global dynamics of the reduced proportional system. If $R_{01} \leq 1$, the disease-free equilibrium $P_{0}$ is globally asymptotically stable. The unique equilibrium $P^{*}$ of the system (2) is globally asymptotically stable in $\stackrel{\circ}{T}$, when $b \geq \alpha$, $\Delta>1, R_{01}>1$, and $\alpha<\min \{\sigma a, b, \varepsilon\}$. When $q=a=\gamma=0$, (2) becomes the SEIR model without infectivity in latent and disease-caused death (see [8]). When $q=0$, (2) becomes the SEIR model without infectious in latent (see [12]).

The parameters are considered in the following cases:

$$
\varepsilon=0.05, \quad \gamma=0.003, \quad \alpha=0.002, \quad \beta=0.05,
$$

(see [16]), and

$$
q=1, \quad b=0.00001, \quad \sigma a=0.000001 .
$$

At this case, there are three fixed points:

$$
\begin{gathered}
E_{1}(0.9091,0,0), \quad E_{2}(0.0911,0.00018673,0.0019), \\
E_{3}(0.0000083926,0.025,24.7973),
\end{gathered}
$$

and $E_{1}, E_{3}$ are unstable, $E_{2}$ stable. The method of direct numerical simulation of (2) shows that there is a periodic solution. The phase portraits of the system (2) in Figure 1(a), and time series of $s, e, i$ are given in Figures 1(b), 1(c), and 1(d).

\section{Acknowledgments}

The authors are very grateful to the reviewers for their valuable comments and suggestions. This work was supported by the Youth Science Foundations of Education Department of Hebei Province (no. 2010233, 2011236).

\section{References}

[1] S. Bowong and J. Kurths, "Parameter estimation based synchronization for an epidemic model with application to tuberculosis in Cameroon," Physics Letters A, vol. 374, no. 44, pp. 4496-4505, 2010.

[2] B. Bokler, "Chaos and complexity in measles models: a comparative numerical study," Mathematical Medicine and Biology, vol. 10, no. 2, pp. 83-95, 1993.

[3] C. T. Bauch, E. Szusz, and L. P. Garrison, "Scheduling of measles vaccination in low-income countries: projections of a dynamic model," Vaccine, vol. 27, no. 31, pp. 4090-4098, 2009.

[4] Z. Mukandavire, C. Chiyaka, W. Garira, and G. Musuka, "Mathematical analysis of a sex-structured HIV/AIDS model with a discrete time delay," Nonlinear Analysis: Theory, Methods and Applications, vol. 71, no. 3-4, pp. 1082-1093, 2009.

[5] H. Huo and L. Feng, "Global stability for an HIV/AIDS epidemic model with different latent stages and treatment," Applied Mathematical Modelling, vol. 37, no. 3, pp. 1480-1489, 2013.

[6] Z. Yang and H. Jia, "Epidemic dynamics model with delay and impulsive vaccination control base on variable population," Mathematical Methods in the Applied Sciences, vol. 34, no. 15, pp. 1822-1832, 2011.
[7] X. Li and B. Fang, "Stability of an age-structured SEIR epidemic model with infectivity in latent period," Applications and Applied Mathematics, vol. 1, pp. 218-236, 2009.

[8] N. Yi, Q. Zhang, K. Mao, D. Yang, and Q. Li, "Analysis and control of an SEIR epidemic system with nonlinear transmission rate," Mathematical and Computer Modelling, vol. 50, no. 9-10, pp. 1498-1513, 2009.

[9] X. Z. Li and L. L. Zhou, "Global stability of an SEIR epidemic model with vertical transmission and saturating contact rate," Chaos, Solitons and Fractals, vol. 40, no. 2, pp. 874-884, 2009.

[10] B. Mukhopadhyay and R. Bhattacharyya, "Analysis of a spatially extended nonlinear SEIS epidemic model with distinct incidence for exposed and infectives," Nonlinear Analysis: Real World Applications, vol. 9, no. 2, pp. 585-598, 2008.

[11] O. Diekmann and J. A. P. Heesterbeek, Mathematical Epidemiology of Infectious Disease, John Wiley and Sons, Chichester, UK, 2000.

[12] C. Sun and Y. H. Hsieh, "Global analysis of an SEIR model with varying population size and vaccination," Applied Mathematical Modelling, vol. 34, no. 10, pp. 2685-2697, 2010.

[13] J. K. Hale, Ordinary Differential Equations, Wiley-Interscience, New York, NY, USA, 1969.

[14] M. Y. Li and J. S. Muldowney, "A geometric approach to globalstability problems," SIAM Journal on Mathematical Analysis, vol. 27, no. 4, pp. 1070-1083, 1996.

[15] R. H. Martin Jr., "Logarithmic norms and projections applied to linear differential systems," Journal of Mathematical Analysis and Applications, vol. 45, no. 2, pp. 432-454, 1974.

[16] E. Demirci, A. Unal, and N. Ozalp, "A fractional order SEIR model with density dependent death rate," Hacettepe Journal of Mathematics and Statistics, vol. 40, no. 2, pp. 287-295, 2011. 


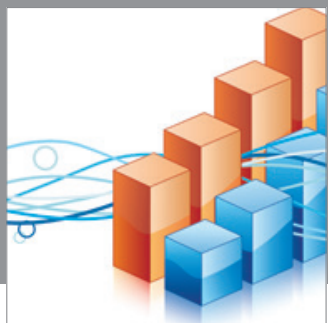

Advances in

Operations Research

mansans

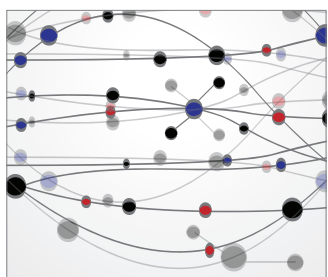

The Scientific World Journal
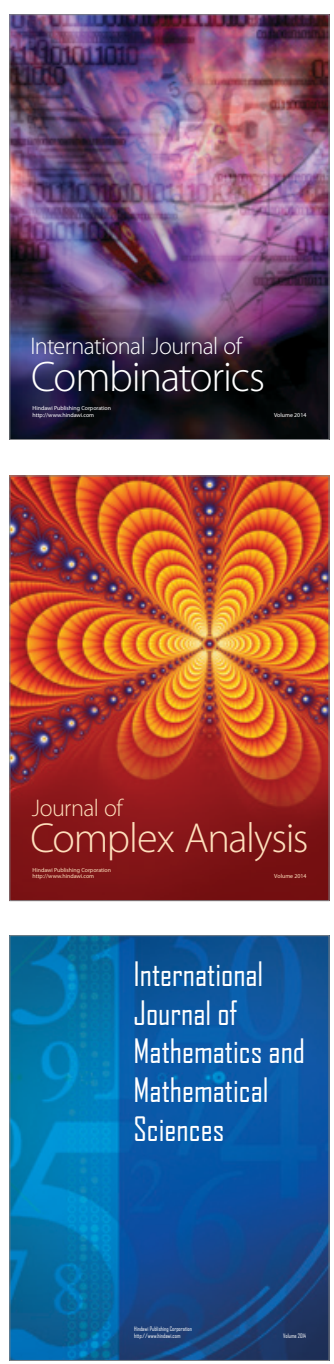
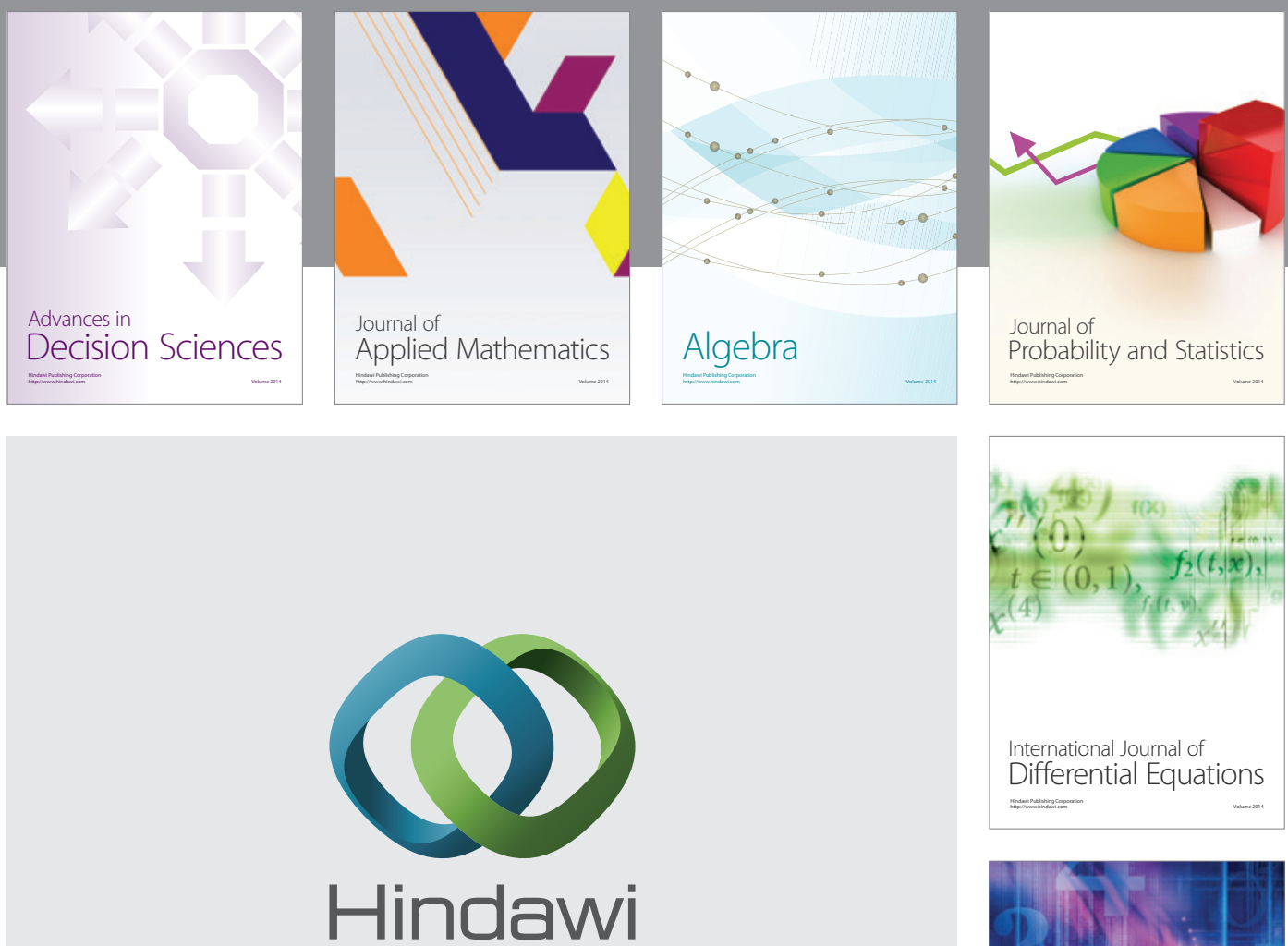

Submit your manuscripts at http://www.hindawi.com
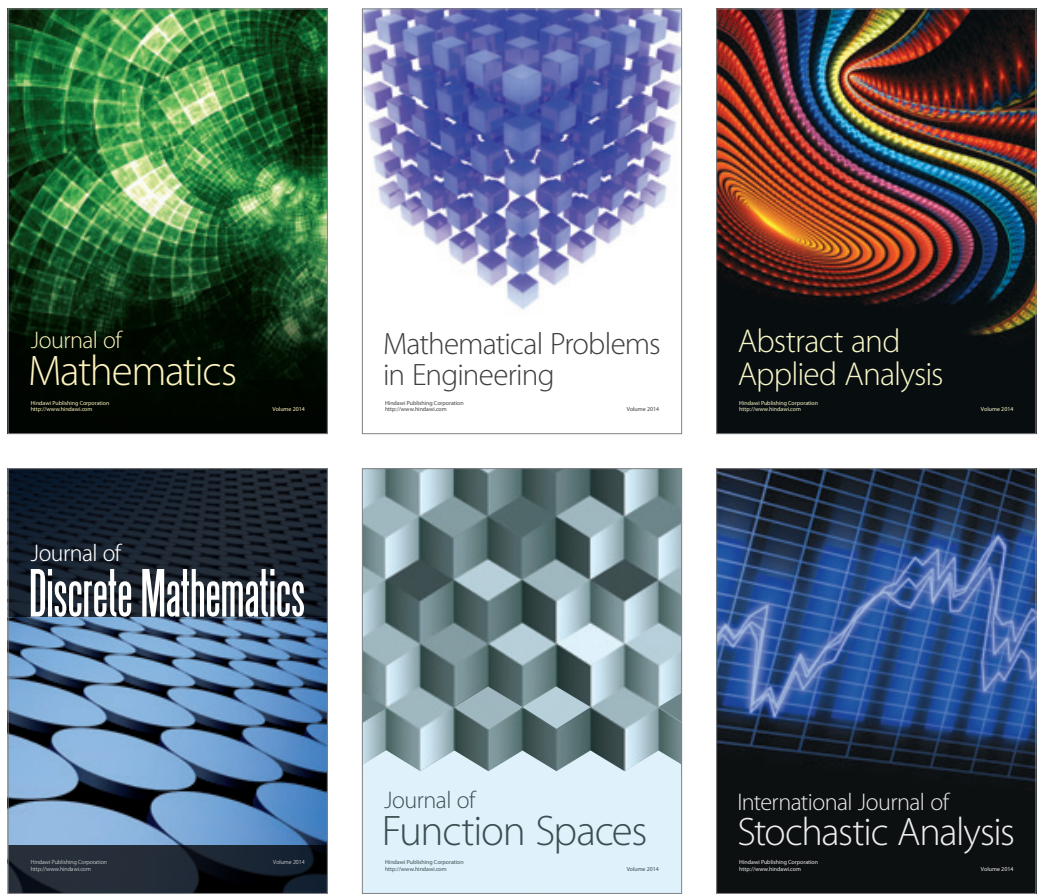

Journal of

Function Spaces

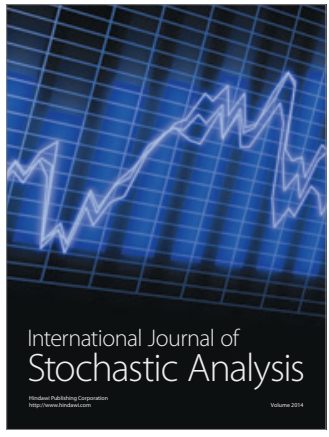

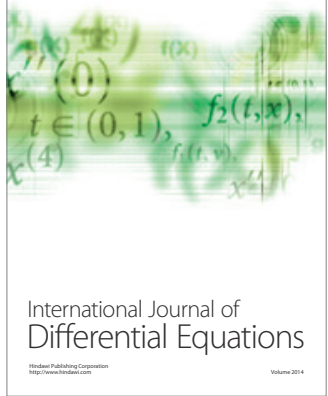
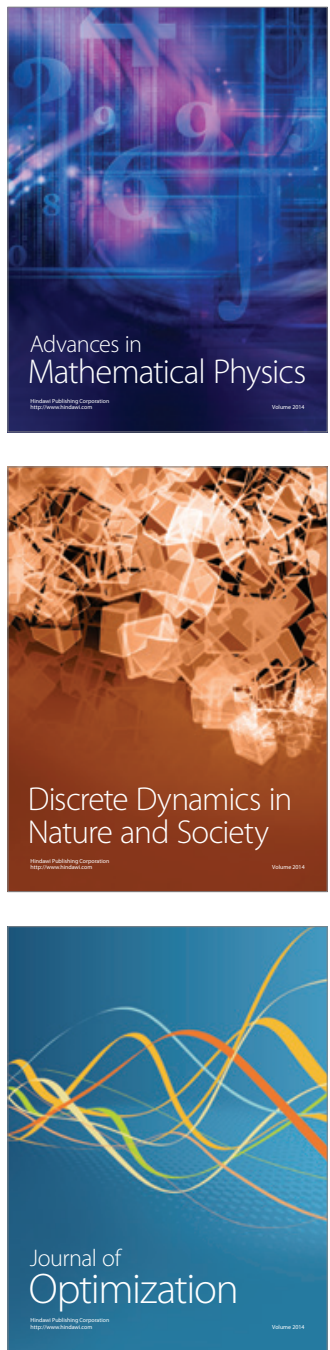